\title{
Entropy of Hybrid Censoring Schemes
}

\author{
H. Morabbi and M. Razmkhah* \\ Ferdowsi University of Mashhad
}

\begin{abstract}
A hybrid censoring scheme is a mixture of type I and type II censoring schemes. When $n$ items are placed on a life test, the experiment terminates under type I or type II hybrid censoring scheme if either a prefixed censoring time $T$ or the $r$ th $(1 \leqslant r \leqslant n$ is fixed) failure is first or later observed, respectively. In this paper, we investigate the decomposition of entropy in both hybrid censoring schemes. Entropy of type I hybrid censoring scheme is formulated and in order to determining the entropy of type II hybrid censoring scheme the available information are used. The results are then applied to some common life time distributions as illustrative examples. Finally, maximum entropy of the mentioned censoring schemes is discussed.

Keywords. Order statistics; entropy change; maximum entropy; exponential distribution; Weibull distribution; Pareto distribution; hazard rate function.
\end{abstract}

\section{Introduction}

In a lifetime experiment, suppose $n$ items are placed on the test for which the observed failure times are the order statistics of a random sample of size $n$ from a distribution with probability density function (pdf) $f(x ; \theta)$ and cumulative distribution function (cdf) $F(x ; \theta)$. The waiting time for the final failure is unbounded, see Muenz and Green (1977), also some items are too expensive. These are the reasons to terminate the experiment before the last failure. In type I censoring scheme, the lifetime experiment is terminated when a pre-fixed censoring time $T$ arrives. In type II censoring scheme, the experiment may instead be terminated when the $r$ th $(1 \leqslant r \leqslant n$ is fixed)

* Corresponding author 
failure is observed. Two mixtures of both these censoring schemes have been extensively discussed in the reliability literature. If the experiment terminates as soon as either the $r$ th failure or the pre-specified censoring time $T$ occurs, type I hybrid censoring scheme has been performed. In type II hybrid censoring scheme, the experiment terminates when the latter of the $r$ th failure and the censoring time $T$ occurs.

Denote the $r$ th order statistic from a random sample of size $n$ by $X_{r: n}$. Thus, in type I hybrid censoring scheme one observes $X_{1: n}, \ldots, X_{s: n}$ when $X_{s: n} \leqslant \min \left\{X_{r: n}, T\right\}$ and $X_{s+1: n}>\min \left\{X_{r: n}, T\right\}$. Under this scheme, the experiment may be terminated too early resulting in very few failures. Under type II hybrid censoring scheme the experiment terminates when $X_{1: n}, \ldots, X_{t: n}$ are observed for which $X_{t: n} \leqslant \max \left\{X_{r: n}, T\right\}$ and $X_{t+1: n}>$ $\max \left\{X_{r: n}, T\right\}$. In both hybrid censoring schemes the failure number $r$ and censoring time $T$ are pre-fixed. Epstein (1954) presented the truncated life tests in the exponential distribution via type I hybrid censored data. Exact inferences for a simple step-stress model with type I and type II hybrid censored data from the exponential distribution are considered by Balakrishnan and Xie (2007 a,b). Kundu (2007) studied the type I and type II hybrid censored Weibull distribution. Park et al. (2008) studied the Fisher information in hybrid censored data. In this paper, we investigate the entropy of hybrid censoring schemes. Entropy measure was introduced into the information theory by Shannon (1948). If $\mathbf{X}=\left(X_{1}, \ldots, X_{n}\right)$ is a continuous random vector with joint pdf $f$, then the entropy of $\mathbf{X}$ is defined as

$$
H_{\mathbf{X}}=-\int_{-\infty}^{\infty} f(\mathbf{x}) \log f(\mathbf{x}) d \mathbf{x},
$$

where $\boldsymbol{x}=\left(x_{1}, \ldots, x_{n}\right)$ is the observed value of $\boldsymbol{X}$. The entropy of an event has been considered by some authors. Wong and Chen (1990) investigated the entropy of ordered sequences and order statistics. Ebrahimi and Soofi (1990) considered relative information loss under type II censored exponential data. Park (1995) studied the entropy of consecutive order statistics. Ebrahimi et al. (2004) also studied the entropy properties of order statistics and spacings.

The main goal of this paper is to study the entropy of hybrid censored data. Toward this end, in Section 2, we first present some basic tools and then we obtain an exact expression for the entropy of type I hybrid censoring scheme. To determine the entropy of type II hybrid censored data, we use the entropy of type I hybrid censoring scheme and type II censored data. In Sec- 
tion 3, applications to some common life time distributions are presented as illustrative examples. Maximum entropy of the mentioned censoring schemes has been discussed in Section 4.

\section{Main Results}

In this section, we study the entropy of hybrid censoring schemes. To this end, we first present some necessary tools. Park (2005) showed that the entropy of type II censored data in a random sample of size $n$ with $r$ failures from a distribution with cdf $F$ and pdf $f$ is

$$
H_{r}^{I I}=\sum_{i=1}^{r}\left\{1-\log (n-i+1)-\int_{-\infty}^{\infty} f_{i: n}(x) \log h(x) d x\right\}
$$

where $h(x)=\frac{f(x)}{1-F(x)}$ is the hazard rate function of $X$ and $f_{i: n}(x)$ is the pdf of $X_{i: n}$.

Hereafter, for more convenience, we denote $a \wedge b$ for $\min \{a, b\}$.

Lemma 1. Let $X_{1}, \ldots, X_{n}$ be a random sample of size $n$ of a random variable with pdf, cdf and hazard rate function $f(x), F(x)$ and $h(x)$, respectively, in which $x>a$, then the entropy of $X_{1: n} \wedge T$ is given by

$$
H_{X_{1: n} \wedge T}(a)=F_{1: n}(T)(1-\log n)-\int_{a}^{T} f_{1: n}(x) \log h(x) d x,
$$

where $F_{1: n}(x)$ and $f_{1: n}(x)$ are the $c d f$ and $p d f$ of $X_{1: n}$, respectively.

Proof. Note that the pdf of $Y=X_{1: n} \wedge T$ is

$$
g_{Y}(y)= \begin{cases}f_{1: n}(y) & a<X_{1: n} \leqslant T, \\ \bar{F}^{n}(T) & X_{1: n}>T .\end{cases}
$$

Therefore, we have

$$
\begin{aligned}
H_{Y}(a) & =E\left[-\log g_{Y}(Y)\right] \\
& =-\int_{a}^{T} f_{1: n}(y) \log f_{1: n}(y) d y-\bar{F}^{n}(T) \log \bar{F}^{n}(T) .
\end{aligned}
$$

By some algebraic calculations, the result follows. 


\subsection{Type I Hybrid Censored Data}

Denote the entropy of type I hybrid censoring scheme by $H_{r}^{h I}$. Let $U_{i}=X_{i: n} \wedge T$ for $i \leqslant r$ and $U_{i}=T$ for $i>r$. To determine $H_{r}^{h I}$, we should calculate the entropy of $U_{1}, \ldots, U_{n}$. First consider the following lemma.

Lemma 2. (Park et al., 2008) The conditional density function of $U_{i}$ given $U_{i-1}=u_{i-1}$ is, for $i \leqslant r$,

$$
f_{i \mid i-1}\left(u_{i} \mid u_{i-1}\right)= \begin{cases}(n-i+1) \frac{f\left(x_{i}\right)}{1-F\left(x_{i-1}\right)}\left\{\frac{1-F\left(x_{i}\right)}{1-F\left(x_{i-1}\right)}\right\}^{n-i} & X_{i: n}=x_{i} \leqslant T, \\ \left\{\frac{1-F(T)}{1-F\left(x_{i-1}\right)}\right\}^{n-i+1} & X_{i: n}=x_{i}>T\end{cases}
$$

and it is obvious that $f_{i \mid i-1}\left(u_{i} \mid u_{i-1}\right)=1$, for $i>r$.

Now, we obtain an expression for $H_{r}^{h I}$ which is stated in the next result.

Theorem 1. Let $X_{1}, \ldots, X_{n}$ be iid continuous random variables with cdf $F(x)$, pdf $f(x)$ and hazard rate function $h(x)$. The entropy of type I hybrid censored sample is given by

$$
\begin{aligned}
H_{r}^{h I}= & \sum_{i=1}^{r}\left[\{1-\log (n-i+1)\}\left\{1-C_{n}^{i-1} \bar{F}^{n-i+1}(T)\right\}\right. \\
& \left.-\int_{-\infty}^{T} f_{i: n}(x) \log h(x) d x\right]
\end{aligned}
$$

where $C_{n}^{i}=\frac{n !}{i !(n-i) !}$.

Proof. By the Markov chain property of order statistics, the joint density function of $U_{1}, \ldots, U_{n}$ can be decomposed as

$$
f_{1, \ldots, n}\left(u_{1}, \ldots, u_{n}\right)=f_{1}\left(u_{1}\right) f_{2 \mid 1}\left(u_{2} \mid u_{1}\right) \cdots f_{r \mid r-1}\left(u_{r} \mid u_{r-1}\right) .
$$

Therefore, the entropy of type I hybrid censored sample can be written as

$$
H_{r}^{h I}=H_{1}+H_{2 \mid 1}+\cdots+H_{r \mid r-1},
$$

where $H_{i \mid i-1}$ is the conditional entropy of $U_{i}$ given $U_{i-1}=u_{i-1}$. By Lemma $2, f_{i \mid i-1}\left(u_{i} \mid u_{i-1}\right)$, for $i \leqslant r$, can be interpreted as the pdf of $Y_{1: n-i+1} \wedge T$ where $Y_{1: n-i+1}$ is the first order statistic of a random sample of size $n-i+1$ 
from a left-truncated random variable with pdf $g(y)=\frac{f(y)}{1-F\left(x_{i-1}\right)}, y>x_{i-1}$, where $x_{i-1}$ is the observed value of $X_{i-1: n}$. Therefore, by Lemma 1 , we have

$$
\begin{aligned}
H_{Y_{1: n-i+1} \wedge T}\left(x_{i-1}\right)= & G_{1: n-i+1}(T)\{1-\log (n-i+1)\} \\
& -\int_{x_{i-1}}^{T} g_{1: n-i+1}(y) \log h_{Y}(y) d y
\end{aligned}
$$

where $G_{1: n-i+1}(y)=1-\left[\frac{1-F(y)}{1-F\left(x_{i-1}\right)}\right]^{n-i+1}$ is the cdf of $Y_{1: n-i+1}, g_{1: n-i+1}(y)$ is the corresponding pdf and $h_{Y}(y)$ is the hazard rate function of $Y$ with pdf $g(y)$. Hence,

$$
\begin{aligned}
H_{i \mid i-1}=H_{Y_{1: n-i+1} \wedge T}= & E\left\{H_{Y_{1: n-i+1} \wedge T}\left(X_{i-1: n}\right)\right\} \\
= & \int_{-\infty}^{\infty} H_{Y_{1: n-i+1} \wedge T}(x) f_{i-1: n}(x) d x \\
= & \{1-\log (n-i+1)\} \\
& \times\left\{1-\bar{F}^{n-i+1}(T) \int_{-\infty}^{\infty} \frac{f_{i-1: n}(x)}{\bar{F}^{n-i+1}(x)} d x\right\} \\
& -(n-i+1) \int_{-\infty}^{T} \int_{-\infty}^{y} f(y) \bar{F}^{n-i}(y) \\
& \times \log \left\{h_{Y}(y)\right\} \frac{f_{i-1: n}(x)}{\bar{F}^{n-i+1}(x)} d x d y .
\end{aligned}
$$

By some algebraic calculations and using the fact that

$$
h_{Y}(y)=\frac{g(y)}{1-G(y)}=\frac{\frac{f(y)}{1-F\left(x_{i-1}\right)}}{\frac{1-F(y)}{1-F\left(x_{i-1}\right)}}=\frac{f(y)}{1-F(y)}=h_{X}(y),
$$

the result follows.

\section{$2.2 \quad$ Type II Hybrid Censored Data}

Denote the entropy of type II hybrid censored data by $H_{r}^{h I I}$. Let $V_{i}=X_{i: n}$ for $i \leqslant r$ and $V_{i}=X_{i: n} \wedge T$ for $i>r$. Here, we calculate the entropy of 
$V_{1}, \ldots, V_{n}$. Notice that for $i \leqslant r$ the conditional density function of $V_{i}$ given $V_{i-1}=v_{i-1}$, is

$$
f_{i \mid i-1}\left(v_{i} \mid v_{i-1}\right)=f_{i \mid i-1: n}\left(x_{i} \mid x_{i-1}\right),
$$

where $f_{i \mid i-1: n}$ is the conditional pdf of $X_{i: n}$ given $X_{i-1: n}=x_{i-1}$. For $i>r$, this conditional density function can be obtained in view of Lemma 2 .

Theorem 2. Under the assumptions of Theorem 1, the entropy of type II hybrid censoring scheme can be determined as follows

$$
H_{r}^{h I I}=H_{r}^{I I}+H_{n}^{h I}-H_{r}^{h I},
$$

where $H_{r}^{I I}$ and $H_{r}^{h I}$ are defined in (1) and (2), respectively.

Proof. By the Markov chain property of order statistics, the joint density function of $V_{1}, \ldots, V_{n}$ can be decomposed as

$$
f_{1, \ldots, n}\left(v_{1}, \ldots, v_{n}\right)=f_{1, \ldots, r}\left(v_{1}, \ldots, v_{r}\right) f_{r+1, \ldots, n \mid r}\left(v_{r+1}, \ldots, v_{n} \mid v_{r}\right),
$$

for which $f_{1, \ldots, r}\left(v_{1}, \ldots, v_{r}\right)$ is the joint pdf of the first $r$ order statistics in a random sample of size $n$, moreover,

$$
f_{r+1, \ldots, n \mid r}\left(v_{r+1}, \ldots, v_{n} \mid v_{r}\right)=f_{r+1 \mid r}\left(v_{r+1} \mid v_{r}\right) \cdots f_{n \mid n-1}\left(v_{n} \mid v_{n-1}\right) .
$$

Therefore, the entropy of type II hybrid censoring scheme is

$$
H_{r}^{h I I}=H_{r}^{I I}+H_{r+1 \mid r}+\cdots+H_{n \mid n-1}=H_{r}^{I I}+\sum_{i=r+1}^{n} H_{i \mid i-1},
$$

where $H_{i \mid i-1}$ is defined in (3). On the other hand, we get

$$
\sum_{i=r+1}^{n} H_{i \mid i-1}=H_{n}^{h I}-H_{r}^{h I}
$$

Hence, the result follows.

\section{Examples}

To illustrate the results of this paper, some common distributions are considered and the entropy of the mentioned censoring schemes is discussed. For each distribution a comparative study has been done. Hereafter, for a parametric family, we denote the entropy of type II censored data, type I and type II hybrid censored samples, cdf, pdf and hazard rate function by $H_{r}^{I I}(\theta), H_{r}^{h I}(\theta), H_{r}^{h I I}(\theta), F(x ; \theta), f(x ; \theta)$ and $h(x ; \theta)$, respectively. 


\subsection{Exponential Distribution}

A random variable $X$ is said to have exponential distribution if its cdf is

$$
F(x ; \theta)=1-e^{-\theta x} \quad x>0, \theta>0 .
$$

Here, we evaluate the entropy of the mentioned censoring schemes in previous sections for the exponential distribution with cdf (4) and investigate the entropy changes in each scheme.

\subsubsection{Type II Censored Sample}

Using (1), the entropy of type II censored sample is

$$
H_{r}^{I I}(\theta)=r(1-\log \theta)-\sum_{i=1}^{r} \log (n-i+1) .
$$

Remark 1 From equation (5), we have

$$
H_{r}^{I I}(\theta)-H_{r-1}^{I I}(\theta)=1-\log \theta-\log (n-r+1) .
$$

Notice that $H_{r}^{I I}(\theta)-H_{r-1}^{I I}(\theta)$ is the entropy change due to adding $X_{r: n}$ to $\left(X_{1: n}, \ldots, X_{r-1: n}\right)$. By $(6)$, the entropy change for type II censored sample in exponential distribution is negative for $r<n+1-\frac{e}{\theta}$ and it is positive for $r>n+1-\frac{e}{\theta}$. That is, the entropy of $\left(X_{1: n}, \ldots, X_{r: n}\right)$ decreases for $r<n+1-\frac{e}{\theta}$ and increases for $r>n+1-\frac{e}{\theta}$ with respect to $r$.

\subsubsection{Type I Hybrid Censored Sample}

Using Theorem 1, the entropy of type I hybrid censored sample is

$$
\begin{aligned}
H_{r}^{h I}(\theta)= & \sum_{i=1}^{r}\left[\{1-\log (n-i+1)\}\left\{1-C_{n}^{i-1} e^{-(n-i+1) \theta T}\right\}\right. \\
& \left.-\log \theta \sum_{j=i}^{n} C_{n}^{j}\left(1-e^{-\theta T}\right)^{j} e^{-(n-j) \theta T}\right] .
\end{aligned}
$$

Remark 2 The entropy change due to adding $U_{r: n}$ to $\left(U_{1: n}, \ldots, U_{r-1: n}\right)$ in type I hybrid censored data is

$$
\begin{aligned}
H_{r}^{h I}(\theta)-H_{r-1}^{h I}(\theta)= & \{1-\log (n-r+1)\}\left\{1-C_{n}^{r-1} e^{-(n-r+1) \theta T}\right\} \\
& -\log \theta \sum_{j=r}^{n} C_{n}^{j}\left(1-e^{-\theta T}\right)^{j} e^{-(n-j) \theta T} .
\end{aligned}
$$


Table 1.The entropy of type II and hybrid (type I and type II) censored sample from the standard exponential distribution with $n=10$ and $T=\log 2$.

\begin{tabular}{ccccccccccc}
\hline \hline$r$ & 1 & 2 & 3 & 4 & 5 & 6 & 7 & 8 & 9 & 10 \\
\hline$H_{r}^{I I}$ & -1.30 & -2.49 & -3.579 & -4.525 & -5.317 & -5.926 & -6.313 & -6.411 & -6.104 & -5.104 \\
$H_{r}^{h I}$ & -1.30 & -2.48 & -3.365 & -3.423 & -1.614 & 2.579 & 7.266 & 8.647 & 5.501 & 1.500 \\
$H_{r}^{h I I}$ & 1.499 & 1.475 & 1.285 & 0.398 & -2.202 & -7.005 & -12.078 & -13.558 & -10.105 & -5.104 \\
\hline
\end{tabular}

For the special case of $\theta=1$, it can be shown that the entropy change is positive for either $\left\{r \mid r>n-e+1\right.$ and $\left.\log C_{n}^{r-1}<(n-r+1) T\right\}$ or $\left\{r \mid r<n-e+1\right.$ and $\left.\log C_{n}^{r-1}>(n-r+1) T\right\}$. For example, if the median of the standard exponential distribution is considered as the pre-fixed censoring time, i.e. $T=\log 2$, the entropy of $\left(U_{1}, \ldots, U_{r}\right)$ decreases for $r \leqslant 4$ or $r \geqslant 9$ and increases for $4<r<9$, when $n=10$.

\subsubsection{Type II Hybrid Censored Sample}

The entropy of type II hybrid censored sample can be easily evaluated using Theorem 2 and equations (5) and (7).

Remark 3 For the special case of $\theta=1$, we have

$$
H_{r}^{h I I}(\theta)-H_{r-1}^{h I I}(\theta)=C_{n}^{r-1} e^{-(n-r+1) T}\{1-\log (n-r+1)\} .
$$

Therefore, the entropy change due to adding $V_{r}$ to $\left(V_{1}, \ldots, V_{r-1}\right)$ does not depend on $\theta$ and it is negative for $r<n+1-e$ and positive for $r>n+1-e$.

To illustrate the results in Remarks 1-3, some numerical values of the entropy of type II and hybrid (type I and type II) censored samples from standard exponential distribution are presented in Table 1 for $n=10, r=1$ up to 10 and pre-fixed censoring time $T=\log 2$.

Figure 1 also shows the entropy of the mentioned censored sample from exponential distribution for $\theta=0.5,1,2, n=10, r=1$ up to 10 and $T=\frac{\log 2}{\theta}$.

Note: In Figures 1, 2 and 3, solid, dot and dash lines show the entropy of type II censored sample, type I and type II hybrid censoring schemes, respectively.

From Figure 1, the following results deduce for exponential distribution:

1. The entropy treatment of three mentioned censoring schemes is similar for $\theta=0.5,1$ and 2 . 
2. For $r \leqslant 5$, the entropy of type II hybrid censoring scheme is more than that for two others, but for $r>5$, type I hybrid censoring scheme seems better.

\subsection{Weibull Distribution}

A random variable $X$ is said to have Weibull distribution if its cdf is

$$
F(x ; \alpha, \beta)=1-e^{-\beta x^{\alpha}} \quad x>0, \alpha>0, \beta>0 .
$$

To perform both hybrid censoring schemes, we use the median of the Weibull distribution (8) as the censoring time, i.e., $T=\left(\frac{\log 2}{\beta}\right)^{\frac{1}{\alpha}}$. Therefore, the entropy of the mentioned censoring schemes are given as follows:

(i) Using (1), the entropy of type II censored samples is

$$
\begin{aligned}
H_{r}^{I I}(\alpha, \beta)= & r\{1-\log (\alpha \beta)\}-\sum_{i=1}^{r}\left[\log (n-i+1)+\frac{\alpha-1}{\alpha} i C_{n}^{i}\right. \\
& \left.\times \sum_{j=0}^{i-1} \frac{(-1)^{j} C_{i-1}^{j}}{n-i+j+1}\{\gamma-\log (\beta(n-i+j+1))\}\right]
\end{aligned}
$$

where $\gamma$ is the Euler's constant.
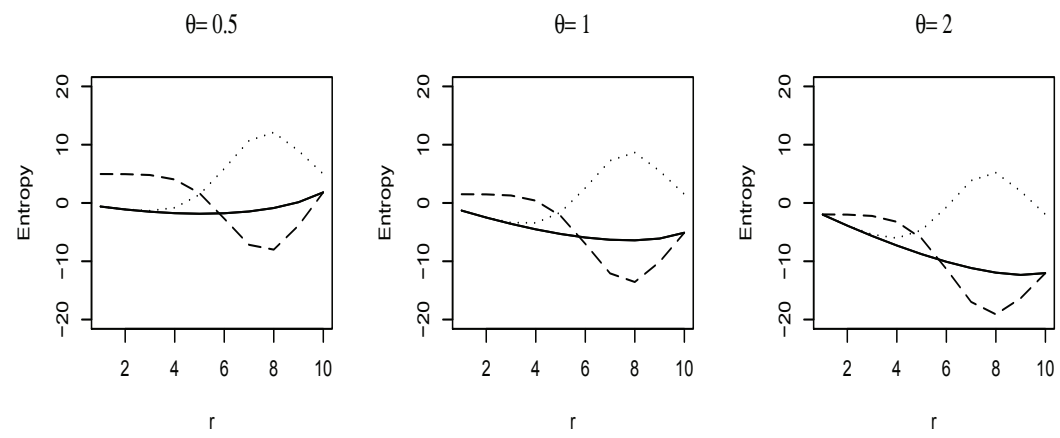

Figure 1. Entropy of censored sample from exponential distribution for $n=10$. 
(ii) By Theorem 1, the entropy of type I hybrid censored data is

$$
\begin{aligned}
H_{r}^{h I}(\alpha, \beta) & =\sum_{i=1}^{r}\left[\{1-\log (n-i+1)\}\left(1-C_{n}^{i-1} 2^{n-i+1}\right)-\log (\alpha \beta) 2^{-n}\right. \\
& \left.\times \sum_{r=i}^{n} C_{n}^{r}-\frac{\alpha-1}{\alpha} \int_{0.5}^{1} i C_{n}^{i}(1-x)^{i-1} x^{n-i} \log \left(\frac{-\log x}{\beta}\right) d x\right] .
\end{aligned}
$$

(iii) The entropy of type II hybrid censored samples can be obtained using Theorem 2.

The amount of entropy of the mentioned censoring schemes are evaluated for $n=10, \alpha=0.5,1,2$ and $\beta=1$. The results are presented in Figure 2 .

From Figure 2, the following results deduce for Weibull distribution:

1. The difference of the entropy treatment of three mentioned censoring schemes for $\theta=0.5,1$ and 2 is not significant.

2. For $r \leqslant 5$ and $r>5$, type II hybrid and type I hybrid censoring schemes have more entropy, respectively.
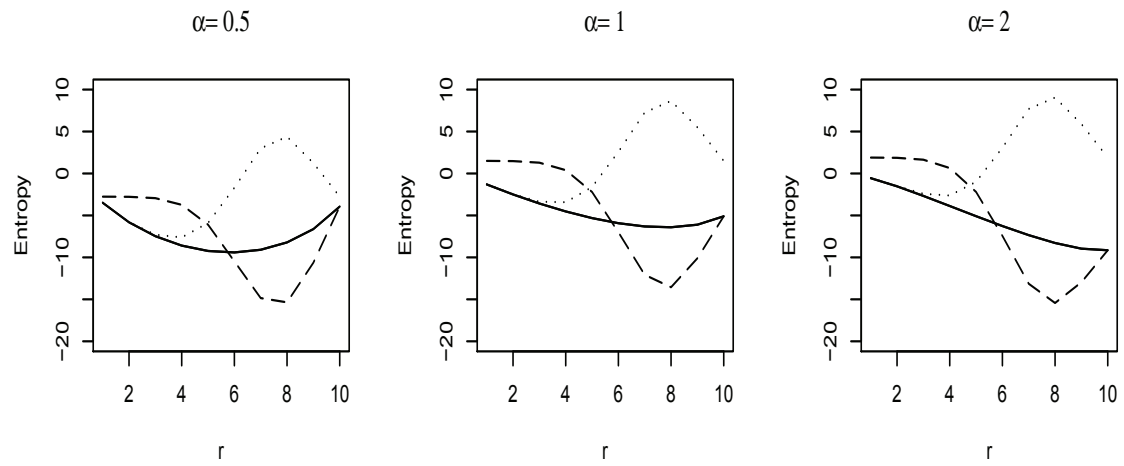

Figure 2. Entropy of censored samples from Weibull distribution for $n=10$. 


\subsection{Pareto Distribution}

A random variable $X$ is said to have Pareto distribution if its cdf is

$$
F(x ; \alpha, \beta)=1-\left(\frac{\beta}{x}\right)^{\alpha} \quad x \geqslant \beta, \alpha>0, \beta>0 .
$$

To apply both hybrid censoring schemes, we consider the censoring time $T=\beta 2^{\frac{1}{\alpha}}$ where is the median of the Pareto distribution (9). Therefore, the entropy of the mentioned censored samples are given as follows:

(i) Using (1), the entropy of type II censoring scheme is

$$
\begin{aligned}
H_{r}^{I I}(\alpha, \beta)= & r\left\{1-\log \left(\frac{\alpha}{\beta}\right)\right\}-\sum_{i=1}^{r}\left\{\log (n-i+1)-\frac{i C_{n}^{i}}{\alpha}\right. \\
& \left.\times \sum_{j=0}^{i-1} \frac{(-1)^{j} C_{i-1}^{j}}{(n-i+j+1)^{2}}\right\} .
\end{aligned}
$$

(ii) By Theorem 1, the entropy of type I hybrid censored data is

$$
\begin{aligned}
H_{r}^{h I}(\alpha, \beta)= & \sum_{i=1}^{r}\left[\{1-\log (n-i+1)\}\left\{1-C_{n}^{i-1} 2^{-(n-i+1)}\right\}\right. \\
& \left.-\log \left(\frac{\alpha}{\beta}\right) 2^{-n} \sum_{r=i}^{n} C_{n}^{r}-\frac{1}{\alpha} \int_{0.5}^{1} i C_{n}^{i}(1-x)^{i-1} x^{n-i} \log x d x\right] .
\end{aligned}
$$

(iii) The entropy of type II hybrid censored samples can be obtained using Theorem 2.

Figure 3 shows the entropy of the mentioned censoring schemes for $n=$ $10, \alpha=0.5,1,2$ and $\beta=1$.

From Figure 3, the following results deduce for Pareto distribution:

1. For $\alpha=0.5$, type II censored sample and type II hybrid censoring scheme have more entropy for $\{r \mid r \leqslant 2$ or $5<r \leqslant 10\}$ and $\{r \mid 2<r \leqslant$ $5\}$, respectively.

2. For $\alpha=1$, type II hybrid censoring scheme, type I hybrid censoring scheme and type II censored sample equally with type II hybrid censoring scheme have more entropy for $r \leqslant 5,\{r \mid 5<r \leqslant 9\}$ and $r=10$, respectively. 
3. For $\alpha=2$, type II and type I hybrid censoring schemes have more entropy for $r \leqslant 4$ and $r>4$, respectively.

\section{Maximization}

In subjectivist probability, the principle of maximum entropy is a postulate which states that subject to known constraints, the probability distribution which best represents the current state of knowledge is the one with largest entropy. We proceed by a class of distributions which has been considered by some authors. Zheng (2001) used this class in a characterization problem by the Fisher information under type II censored samples, Ahmadi and Arghami (2001) studied it to compare the record statistics and iid observations in view of Fisher information and Baratpour et al. (2007) characterize this class by the entropy of the record values.

Let $C$ be a class of distributions $F$ of non-negative random variables $X$ with $F(0)=0$ such that

$$
\begin{gathered}
h(x ; \theta)=\alpha(\theta) b(x), \\
b(x) \geqslant M>0,
\end{gathered}
$$

where $h(x ; \theta)$ is the hazard rate function of $X, b(x)$ is the first derivative of $B(x)$, an increasing function on positive real line with $B(0)=0$ and $\alpha(\theta)$ is a non-negative function of $\theta$.

$\alpha=0.5$

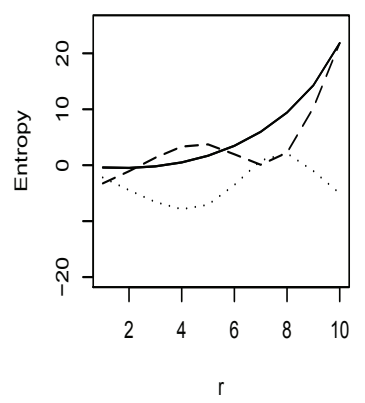

$\alpha=1$

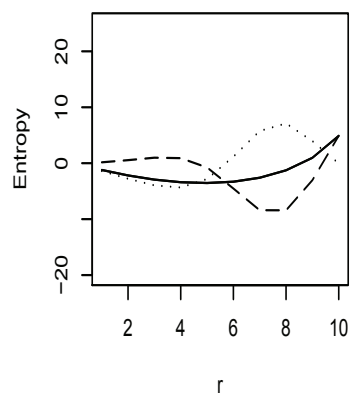

$\alpha=2$

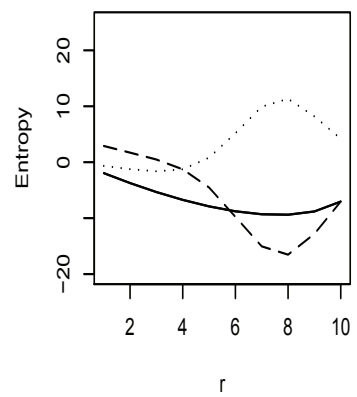

Figure 3. Entropy of censored sample from Pareto distribution for $n=10$. 
Consider the set of all trial probability distributions that belong to $C$. In this section, we derive the probability distribution that maximizes the entropy of the mentioned censoring schemes in previous sections with respect to the constraints (10) and (11). First we recall the following lemma which is a corollary of Stone-Weierstrass Theorem (see Aliprantis and Burkinshaw, 1981).

Lemma 3. If $g$ is a continuous function on [0,1] such that $\int_{0}^{1} x^{n} g(x) d x=0$ for $n \geqslant 0$, then $g(x)=0$ for all $x \in[0,1]$.

In the next results we show that the exponential distribution maximizes the entropy of the mentioned censoring schemes.

Theorem 3. Type II censored sample of the distribution $F$ has maximum entropy in $C$, if and only if $F(x ; \theta)=1-e^{-M \alpha(\theta) x}$.

Proof. By (10), it can be shown that if $F$ belongs to $C$, then

$$
F(x ; \theta)=1-e^{-\alpha(\theta) B(x)} .
$$

(Necessity) Using (1), (10) and (11), we have

$$
\begin{aligned}
H_{r}^{I I}(\theta)= & \sum_{i=1}^{r}[1-\log (n-i+1)-\log \alpha(\theta) \\
& \left.-\int_{0}^{1} i C_{n}^{i} x^{i-1}(1-x)^{n-i} \log \left\{b\left(B^{-1}\left(\frac{-1}{\alpha(\theta)} \log (1-x)\right)\right)\right\} d x\right] \\
\leqslant & \sum_{i=1}^{r}\{1-\log (n-i+1)-\log \alpha(\theta)-\log M\} .
\end{aligned}
$$

The right hand side of the inequality (13) is the entropy of the type II censored sample of distribution $F(x ; \theta)=1-e^{-M \alpha(\theta) x}$. Therefore, since the entropy of the order statistics in a random sample characterizes the parent distribution (see, Baratpour et al., 2007), the type II censored sample of this distribution has maximum entropy.

(Sufficiency) Let the type II censored sample of the distribution $F$ have maximum entropy in $C$. Therefore, by (13) we have

$$
\int_{0}^{1} x^{i-1}(1-x)^{n-i} \log \left\{\frac{1}{M} b\left(B^{-1}\left(\frac{-1}{\alpha(\theta)} \log (1-x)\right)\right)\right\} d x=0 .
$$


Using Lemma 3, we can deduce

$$
\log \left\{\frac{1}{M} b\left(B^{-1}\left(\frac{-1}{\alpha(\theta)} \log (1-x)\right)\right)\right\}=0
$$

or equivalently,

$$
\frac{d}{d x} B^{-1}\left\{\frac{-1}{\alpha(\theta)} \log (1-x)\right\}=\frac{1}{M \alpha(\theta)(1-x)} .
$$

Hence,

$$
B^{-1}\left\{\frac{-1}{\alpha(\theta)} \log (1-x)\right\}=\frac{-1}{M \alpha(\theta)} \log (1-x)+g(\theta),
$$

where by noting that $B(0)=0$, we get $g(\theta)=0$. So, by taking $y=$ $\frac{-1}{\alpha(\theta)} \log (1-x)$, we have $B^{-1}(y)=\frac{y}{M}$ and then $B(x)=M x$. Thus by (12), the result follows.

Theorem 4. Type I hybrid censored sample of the distribution $F$ has maximum entropy in $C$, if and only if $F(x)=1-e^{-M \alpha(\theta) x}$.

The proof of the Theorem 4 is similar to that of Theorem 3, therefore it is omitted.

Corollary 1 According to Theorems 2, 3 and 4, Type II hybrid censored sample of the distribution $F$ has maximum entropy in $C$, if and only if $F(x)=1-e^{-M \alpha(\theta) x}$.

\section{Acknowledgement}

The authors would like to thank the referees for their useful comments and careful reading the manuscript that definitely improved the paper. Partial support from the Ordered and Spatial Data Center of Excellence of Ferdowsi University of Mashhad is acknowledged.

\section{References}

Ahmadi, J. and Arghami, N.R. (2001). On the Fisher information in record values. Metrika, 53, 195-206.

Aliprantis, C.D. and Burkinshaw, O. (1981). Principles of Real Analysis. Elsevier North Holland Inc. 
Balakrishnan, N. and Xie, Q. (2007 a). Exact inference for a simple step-stress model with Type-I hybrid censored data from the exponential distribution. J. Statist. Plann. Inference, 137, 3268-3290.

Balakrishnan, N. and Xie, Q. (2007 b). Exact inference for a simple step-stress model with Type-II hybrid censored data from the exponential distribution. J. Statist. Plann. Inference, 137, 2543-2563.

Baratpour, S., Ahmadi, J. and Arghami, N.R. (2007). Some characterizations based on entropy of order statistics and record values. Comm. Statist. Theo. Meth., 36, 47-57.

Ebrahimi, N. and Soofi, E. (1990). Relative information loss under Type II censored exponential data. Biometrika, 77, 429-435.

Ebrahimi, N., Soofi, E. and Zahedi, H. (2004). Information properties of order statistics and spacings. IEEE, Trans. Inform. Theory, 50, 177-183.

Epstein, B. (1954). Truncated life tests in the exponential case. Ann. Math. Statist., 25, $555-564$.

Kundu, D. (2007). On hybrid censored Weibull distribution. J. Statist. Plann. Inference, 137, $2127-2142$.

Muenz, L.R. and Green, S.B. (1977). Time saving in censored life testing. J. R. Statist. Assoc., Ser. B, 39, 269-275.

Park, S. (1995). The entropy of consecutive order statistics. IEEE, Trans. Inform. Theory, 41, 2003-2007.

Park, S. (2005). Testing exponentiality based on the Kullback-Leibler information with the type II censored data. IEEE, Trans. Rel., 54, 22-26.

Park, S., Balakrishnan, N. and Zheng, G. (2008). Fisher information in hybrid censored data. Statist. Probab. Lett., 78, 2781-2786.

Shannon, C.E. (1948). A mathematical theory of communication. Bell Syst. Tech. J., 27, 379-423.

Wong, K.N. and Chen SH. (1990). The entropy of ordered sequences and order statistics. IEEE Trans. Inform. Theory, 36, 276-284.

Zheng, G. (2001). A characterization of the factorization of hazard function by the Fisher information under Type II censoring with application to the Weibull family. Statist. Probab. Lett., 52, 249 -253. 


\section{H. Morabbi}

Department of Statistics,

Ferdowsi University of Mashhad,

P. O. Box 91775-1159,

Mashhad, Iran.

email:h.morabbi@gmail.com

\section{Razmkhah}

Department of Statistics,

Ferdowsi University of Mashhad,

P. O. Box 91775-1159,

Mashhad, Iran.

email: razmkhah_m@um.ac.ir 